\title{
Unexpected properties of the first excited state of non-bipartite Heisenberg spin rings
}

\author{
J. Schnack \\ Universität Osnabrück, Fachbereich Physik \\ Barbarastr. 7, 49069 Osnabrück, Germany
}

\begin{abstract}
Systematic properties of the first excited state are presented for various ring sizes and spin quantum numbers which are only partly covered by the theorem of Lieb, Schultz and Mattis. For odd ring sizes the first excited energy eigenvalue shows unexpected degeneracy and related shift quantum numbers. As a byproduct the ground state energy as well as the energy of the first excited state of infinite chains are calculated by extrapolating the properties of only a few, finite, antiferromagnetically coupled Heisenberg rings using the powerful Levin sequence acceleration method.
\end{abstract}

PACS: 75.10.Jm, 75.40.Cx

\section{INTRODUCTION AND SUMMARY}

Exact diagonalization methods [1 5] make it possible to investigate small spin rings for various numbers $N$ of spin sites and spin quantum numbers $s$, for instance in the Heisenberg model. The symmetries of the isotropic Heisenberg Hamilton operator allow to decompose the Hilbert space $\mathcal{H}$ into a set of mutually orthogonal subspaces $\mathcal{H}(S, M, k)$ according to the quantum numbers of the total spin $S$, the total magnetic quantum number $M$ and the translational quantum number $k$ of the cyclic shift operator. Since these subspaces are much smaller, a complete or partial diagonalization of the Hamilton matrix is feasible.

The interest in the Heisenberg model, which is known already for a long time, was renewed by the successful synthesis of small magnetic molecules, among them nearly perfect ring structures of paramagnetic ions like $\mathrm{Fe}^{3+}[6]$. One can say that in the majority of these molecules the ions couple antiferromagnetically and the spectrum is rather well described by the Heisenberg model with isotropic next neighbour interaction.

Looking at the properties of the calculated spin rings one realizes that not only the ground states, but also the first excited states share systematic properties which are understood only for Heisenberg spin rings of an even number of spin sites (bipartite systems), i.e. they can in part be derived from the theorem of Lieb, Schultz and Mattis [9.10]. Non-bipartite rings which have an odd number of sites, and thus can be characterized as frustrated, show unexpected properties in degeneracy and translational quantum number.

Knowing systematic rules for quantum numbers also of non-bipartite systems would be very useful for comparison of theoretical results with measurements in ESR/EPR, torque magnetometry or neutron scattering, see e.g. [1,11]. One could employ knowledge about 
quantum numbers of ground and first excited states in order to understand the thermal behaviour of quantities like the magnetic susceptibility. In addition these exact values may help to improve low temperature approximations. Usually the high temperature behaviour of observables is well known, e.g. from classical spin dynamics [12], but at low temperature such approximations are poor. The knowledge of ground and first excited states could already be sufficient for a considerable improvement.

Having evaluated the spectra of small Heisenberg rings with isotropic next-neighbour interaction one can approximate the infinite chain limit, which for the $s=\frac{1}{2}$ ground state is known as the Bethe-Hulthén limit [13,[14]. Because the sequences converge rather slowly and only a limited number of energy eigenvalues can be evaluated, the Levin $u$-sequence acceleration method [15,16] is employed, which leads to impressive estimates of the antiferromagnetic ground state energies as well as of the excitation gap for infinite rings or chains of larger spin quantum numbers.

\section{SYSTEMATIC PROPERTIES OF THE SPECTRUM}

The Hamilton operator of the Heisenberg model with antiferromagnetic, isotropic next neighbor interaction between spins of equal spin quantum number $s$ is given by

$$
\underset{\sim}{H}=-2 J \sum_{x=1}^{N} \underset{\sim}{\vec{S}}(x) \cdot \underset{\sim}{\vec{S}}(x+1), \quad \forall x: s(x)=s, \quad J<0, \quad N+1 \equiv 1 .
$$

$\underset{\sim}{H}$ commutes with the total spin $\underset{\sim}{\vec{S}}$ and its three-component $\underset{\sim}{S}$. In addition $\underset{\sim}{H}$ is invariant under cyclic shifts generated by the cyclic shift operator $\underset{\sim}{T} . \underset{\sim}{T}$ is defined by its action on the product basis (3)

$$
\underset{\sim}{T}\left|m_{1}, \ldots, m_{N-1}, m_{N}\right\rangle=\left|m_{N}, m_{1}, \ldots, m_{N-1}\right\rangle,
$$

where the product basis is constructed from single-particle eigenstates of all ${\underset{\sim}{3}}^{3}(x)$

$$
\stackrel{\sim}{s}^{3}(x)\left|m_{1}, \ldots, m_{x}, \ldots, m_{N}\right\rangle=m_{x}\left|m_{1}, \ldots, m_{x}, \ldots, m_{N}\right\rangle .
$$

The translational quantum number $k=0, \ldots, N-1$ modulo $N$ labels the eigenvalues of $\underset{\sim}{T}$ which are the $N$-th roots of unity

$$
z=\exp \left\{-i \frac{2 \pi k}{N}\right\}
$$

Exact diagonalization methods [5, [1] allow to evaluate eigenvalues and eigenvectors of $\underset{\sim}{H}$ for small spin rings of various numbers $N$ of spin sites and spin quantum numbers $s$. Systematic investigations [17 21] revealed interesting properties of ground state quantum numbers, compare table II, which only for bipartite rings can be explained by the theorem of Lieb, Schultz and Mattis [9, 10]. The ground state spin quantum number and the degeneracy, for example, depend solely on $N \cdot s$. If $N \cdot s$ is integer, then the ground state has $S=0$ and is non-degenerate; if $N \cdot s$ is half integer, then the ground state has $S=1 / 2$ and is fourfold degenerate [21]. 
It appears that for the properties of the first excited state such rules do not hold in general, but only for "high enough" $N(N>5)$. Then, as can be anticipated from table [1, we can conjecture that

- if $N$ is even, then the first excited state has $S=1$ and is threefold degenerate, and

- if $N$ is odd and the single particle spin is half-integer, then the first excited state has $S=3 / 2$ and is eightfold degenerate, whereas

- if $N$ is odd and the single particle spin is integer, then the first excited state has $S=1$ and is sixfold degenerate.

Except for small odd $N$ also the cyclic shift quantum numbers $k$ of the first excited state show an interesting regularity.

- For odd $N \geq 7, k$ assumes a certain value for all integer spins and another value for all half-integer spins. We conjecture that the $k$ quantum numbers for half-integer spins are $k=3\lfloor(N+1) / 4\rfloor$ and $k=N-3\lfloor(N+1) / 4\rfloor .\lfloor(N+1) / 4\rfloor$ symbolizes the greatest integer less or equal to $(N+1) / 4$. For integer spins numerical data are poor but it seems that $k$ is as close as possible to $N / 2$, i.e. $k=\lfloor N / 2\rfloor$ and $k=N-\lfloor N / 2\rfloor$.

- For even $N$ the shift quantum number $k$ is $N / 2$, if $N / 2$ is also even; if $N / 2$ is odd, $k=0$ for half-integer spin and $k=N / 2$ for integer spin.

For spin- $\frac{1}{2}$-rings these properties may be also derived using the Bethe ansatz [13, 14.

\section{INFINITE CHAIN LIMITS}

Besides the importance of the above presented results for magnetic molecules 11,22, 27,23, 11, 24, the obtained energy eigenvalues enable us to estimate the antiferromagnetic ground state energy $E_{0}(N)$ in the large $N$ limit for a variety of spin quantum numbers. Of course this calculation cannot compete with nowadays DMRG results, but reaches astonishingly close.

As one can see in table [ or Fig. [ (1.h.s.) the convergence of energy eigenvalues with $N$ is rather slow. Therefore, an improved estimate is calculated using the Levin $u$-sequence acceleration method [15], which is appropriate if the series elements form an approximately linear function in $\frac{1}{i^{k}}$ with a certain positive $k$, which to first approximation is the case for

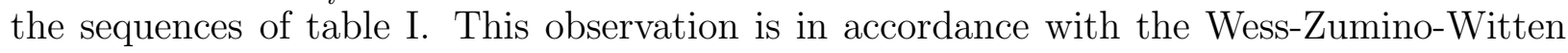
model, see e.g. [25], which yields

$$
\frac{E_{0}}{N} \approx \epsilon_{\infty}-\frac{\alpha}{N^{2}}
$$

Let us denote the elements of our series by $U_{1}, U_{2}, U_{3}, \ldots$ In order to construct monotonic sequences the alternating series for a certain spin are divided into two monotonic subseries

e.g. for $s=\frac{1}{2}$ : $U_{1}=E_{0}(N=2), U_{2}=E_{0}(N=4), U_{3}=E_{0}(N=6), \ldots$ The differences between successive sequence elements are labeled $u_{1}=U_{1}, u_{2}=U_{2}-U_{1}, u_{3}=U_{3}-U_{2}, \ldots$ Then the Levin $u$-estimate using $n$ series elements reads 


$$
U[n]=\frac{\sum_{k=1}^{n}(-1)^{k-1}\left(\begin{array}{l}
n \\
k
\end{array}\right) k^{n-2} \frac{U_{k}}{u_{k}}}{\sum_{k=1}^{n}(-1)^{k-1}\left(\begin{array}{l}
n \\
k
\end{array}\right) k^{n-2} \frac{1}{u_{k}}} .
$$

The following example for the sequence built of the energies for even numbers of spin $s=1 / 2$ demonstrates how fast the Levin $u$-estimate approaches the correct limit

$$
\begin{aligned}
\{U[n]\}= & \{1.5,1.2,0.8735510038,0.8885066176,0.8858640679,0.8863719562, \\
& 0.8862817068,0.8862961998,0.8862941347, \ldots\} \rightarrow 0.8862943611=2 \ln 2-\frac{1}{2} .
\end{aligned}
$$

Roughly one can say that, if the deviations from a power-law behaviour are small, $n$ sequence elements lead to an accuracy of about $n-4$ digits, see Fig. 目 (r.h.s.). Of course the limit can only be as accurate as the individual sequence elements.

The procedure is also applied to spin values $s=1,3 / 2,2,5 / 2$ and the results are depicted in Fig. 2. The hatched areas indicate the interval according to the gained accuracy of the sequence acceleration method, the attached number denotes the mean value of the interval. Since the sequences built from values for even $N$ converge much faster, they determine the results.

In the upper left of Fig. 2 2 the estimate for the infinite chain limit for spin $s=1$ is shown. The estimated ground state energy of $E_{0} /(N J)=2.802967 \pm 0.0000005$ agrees very well with the result 2.802968077942(4) found in Ref. [30] and also with other DMRG and TMRG calculations, see e.g. [31.32]. Also for $s=3 / 2$ the limit is rather well approximated, the value of $E_{0} /(N J)=5.65681 \pm 0.00001$ suggests new discussion of the results $5.65666 \pm 0.00002$ of Ref. [33] and 5.658 of Ref. [32]. For these sequences previously obtained exact diagonalization results have been used, too [26 29.31].

The excitation energies of the first excited state, see table [, enable us to approach the gap for infinite chains or rings. It vanishes for half integer spins and remains finite for integer

spins, Haldane conjecture [34,35]. The following example shows the Levin $u$-sequence for $s=1 / 2$ and even $N$

$$
\begin{aligned}
\{U[n]\}= & \{4 ., 3 .,-2.464274955,0.26749212,0.05231106824,-0.04294415611, \\
& 0.01234278872,-0.003416797715,0.0001536344576363, \ldots\} \rightarrow 0 .
\end{aligned}
$$

In Fig. 3 one can see that the behaviour of the sequence is much smoother for even $N$, whereas the somewhat strange behaviour for small odd $N$ destroys a fast convergence. The convergence of the gap sequences is slowed down by much stronger logarithmic corrections to the power-law behaviour than present in the ground state energy sequences. Thus the gained accuracy for higher spin quantum numbers is rather limited and larger rings together with methods like DMRG have to be used, see e.g. [31.37.38].

\section{ACKNOWLEDGMENTS}

The author would like to thank M. Luban for introducing the Levin methods to him and K. Bärwinkel, E. Kotomin, D. Mentrup and H.-J. Schmidt for helpful discussions. 


\section{REFERENCES}

* author: jschnack@uos.de, http://www.physik.uni-osnabrueck.de/makrosysteme/

[1] A. Bencini, D. Gatteschi, Electron parametric resonance of exchange coupled systems, Springer, Berlin, Heidelberg (1990)

[2] D. Kouzoudis, Heisenberg $s=\frac{1}{2}$ ring consisting of a prime number of atoms, J. Magn. Magn. Mater. 173, 259 (1997)

[3] D. Kouzoudis, Exact analytical partition function and energy levels for a Heisenberg ring of $N=6$ spin 1/2 sites, J. Magn. Magn. Mater. 189, 366 (1998)

[4] O. Waldmann Symmetry and energy spectrum of high-nuclearity spin clusters, Phys. Rev. B 61, 6138 (2000)

[5] K. Bärwinkel, H.-J. Schmidt, J. Schnack, Structure and relevant dimension of the Heisenberg model and applications to spin rings, J. Magn. Magn. Mater. 212, 240 (1999)

[6] K.L. Taft, C.D. Delfs, G.C. Papaefthymiou, S. Foner, D. Gatteschi, and S.J. Lippard, $\left[\mathrm{Fe}(\mathrm{OMe})_{2}\left(\mathrm{O}_{2} \mathrm{CCH}_{2} \mathrm{Cl}\right)\right]_{10}$, a molecular ferric wheel, J. Am. Chem. Soc. 116, 823 (1994)

[7] A. Caneschi, A. Cornia, A.C. Fabretti, S. Foner, D. Gatteschi, R. Grandi, and L. Schenetti, Synthesis, crystal structure, magnetism, and magnetic anisotropy of cyclic clusters comprising six iron(III) ions and entrapping alkaline ions, Chem. Eur. J. 2, 1379 (1996)

[8] A. Lascialfari, D. Gatteschi, F. Borsa, and A. Cornia, Spin dynamics in mesoscopic size magnetic systems: $A^{1} H$ NMR study of iron (III) ions, Phys. Rev. B 55, 14341 (1997)

[9] E.H. Lieb, T.D. Schultz, D.C. Mattis, Antiferromagnetic chain, Ann. Phys. (N.Y.) 16, 407 (1961)

[10] E.H. Lieb, D.C. Mattis, Ordering energy levels of interacting spin systems, J. Math. Phys. 3, 749 (1962)

[11] O. Waldmann, J. Schülein, R. Koch, P. Müller, I. Bernt, R. W. Saalfrank, H.P. Andres, H.U. Güdel, P. Allenspach, Magnetic anisotropy of two cyclic hexanuclear Fe(III) clusters entrapping alkaline ions, Inorg. Chem. 38, 5879 (1999)

[12] J. H. Luscombe, M. Luban, F. Borsa, Classical Heisenberg model of magnetic ring clusters: Accurate approximations for correlation functions and susceptibility, J. Chem. Phys. 108, 7266 (1998)

[13] H. Bethe, Z. Phys. 71, 205 (1931)

[14] L. Hulthén, Arkiv Mat. Astron. Fysik 26A, No. 11 (1938)

[15] D. Levin, Development of non-linear transformations for improving convergence of sequences, Intern. J. Computer Math. B 2, 371 (1973)

[16] M. Luban, Acceleration of convergence of the 1/d expansion for the critical temperature of the spherical model, Prog. Theor. Phys. 58, 1177 (1977)

[17] J.C. Bonner, M.E. Fisher, Linear magnetic chains with anisotropic coupling, Phys. Rev. 135, A640 (1964)

[18] R. Botet, R. Jullien, Ground-state properties of a spin-1 antiferromagnetic chain, Phys. Rev. B 27, 613 (1983)

[19] K. Fabricius, U. Löw, K.-H. Mütter, P. Ueberholz, Complete Solution of the antiferromagnetic Heisenberg rings with $N=12-16$ sites, Phys. Rev. B 44, 7476 (1991)

[20] M. Karbach, Finite-Size-Effekte im eindimensionalen Spin- $\frac{1}{2}-X X Z$-Modell, PhD thesis, Bergische Universität - Gesamthochschule Wuppertal (1994), WUB-DIS 94-6 
[21] K. Bärwinkel, H.-J. Schmidt, J. Schnack, Ground state properties of antiferromagnetic Heisenberg spin rings, J. Magn. Magn. Mater. (2000) accepted

[22] C. Delfs, D. Gatteschi, L. Pardi, R. Sessoli, K. Wieghardt, D. Hanke, Magnetic properties of an octanuclear iron(III) cation, Inorg. Chem. 32, 3099 (1993)

[23] B. Pilawa, R. Desquiotz, M.T. Kelemen, M. Weickenmeier, A. Geisselman, Magnetic properties of new $\mathrm{Fe}_{6}$ (triethanolaminate(3-)) 6 spin clusters, J. Magn. Magn. Mater. 177, 748 (1997)

[24] Y. Furukawa, M. Luban, A. Bino, F. Borsa, D.C. Johnston, A.V. Mahajan, D. Mentrup, L.L. Miller, J. Schnack, Spin dynamics of the magnetic cluster

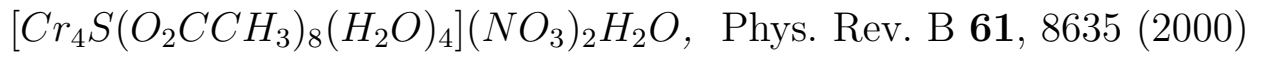

[25] I. Affleck, D. Gepner, H.J. Schulz, T. Ziman, J. Phys. A 22, 511 (1989)

[26] U. Glaus, T. Schneider, Phys. Rev. B 30, 215 (1984)

[27] J.B. Parkinson, J.C. Bonner, Phys. Rev. B 32, 4703 (1985)

[28] A. Moreo, Phys. Rev. B 35, 8562 (1987)

[29] H.Q. Lin, Phys. Rev. B 42, 6561 (1990)

[30] S.R. White, D.A. Huse, Numerical renormalization-group study of low-lying eigenstates of the antiferromagnetic $s=1$ Heisenberg chain, Phys. Rev. B 48, 3844 (1993)

[31] O. Golinelli, Th. Jolicœur, R. Lacaze, Finite-lattice extrapolations for a Haldane gap antiferromagnet, Phys. Rev. B 50, 3037 (1994)

[32] Tao Xiang, Thermodynamics of quantum Heisenberg spin chains, Phys. Rev. B 58, 9142 (1998) and private communication

[33] K. Hallberg, X.Q.G. Wang, P. Horsch, A. Moreo, Critical behavior of the $S=3 / 2$ antiferromagnetic Heisenberg chain, Phys. Rev. Lett. 76, 4955 (1996)

[34] F.D.M. Haldane, Phys. Lett. 93A, 464 (1983)

[35] F.D.M. Haldane, Phys. Rev. Lett. 50, 1153 (1983)

[36] E. Manousakis, Rev. Mod. Phys. 63, 1 (1991)

[37] U. Schollwöck, O. Golinelli, Th. Jolicœur, S=2 antiferromagnetic quantum spin chain, Phys. Rev. B 54, 4038 (1996)

[38] Xiaoqun Wang, Shaojing Qin, Lu Yu Haldane gap for the $S=2$ antiferromagnetic Heisenberg chain revisited, Phys. Rev. B 60, 14529 (1999) 
TABLES

\begin{tabular}{|c|c|c|c|c|c|c|c|c|c|c|}
\hline \multirow[t]{2}{*}{$s$} & \multicolumn{9}{|c|}{$N$} & \\
\hline & 2 & 3 & 4 & 5 & 6 & $7 \mid$ & 8 & 9 & 10 & \\
\hline \multirow{4}{*}{$\frac{1}{2}$} & \begin{tabular}{|c|}
1.5 \\
\end{tabular} & (0.5 & $\mid \overline{11}$ & $\mid \overline{\mid c .747}$ & $\mid$ & 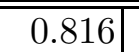 & $\mid \overline{|c| 913}$ & $\mid$ & $\mid \overline{|c| 903}$ & $E_{0} /(N J)$ \\
\hline & 1 & 4 & 1 & 4 & 1 & 4 & 1 & 4 & 1 & $\operatorname{deg}$ \\
\hline & 0 & $1 / 2$ & 0 & $1 / 2$ & 0 & $1 / 2$ & 0 & $1 / 2$ & 0 & $S$ \\
\hline & 1 & 1,2 & 0 & 1,4 & 3 & 2,5 & 0 & 2,7 & 5 & $k$ \\
\hline \multirow{4}{*}{$\frac{1}{2}$} & 4.0 & \begin{tabular}{l|l}
3.0 \\
\end{tabular} & 2.0 & 2.236 & 1.369 & 2.098 & 1.045 & \begin{tabular}{|l|}
1.722 \\
\end{tabular} & 0.846 & $\overline{\Delta E /|J|}$ \\
\hline & 3 & 4 & 3 & 2 & 3 & 8 & 3 & 8 & 3 & $\operatorname{deg}$ \\
\hline & 1 & $3 / 2$ & 1 & $1 / 2$ & 1 & $3 / 2$ & 1 & $3 / 2$ & 1 & $S$ \\
\hline & 0 & 0 & 2 & 0 & 0 & 1,6 & 4 & 3,6 & 0 & $k$ \\
\hline \multirow{4}{*}{1} & 4 & 2 & 3 & 2.612 & 2.872 & 2.735 & 2.834 & 2.773 & 2.819 & $E_{0} /(N J)$ \\
\hline & 1 & 1 & 1 & 1 & 1 & 1 & 1 & 1 & 1 & deg \\
\hline & 0 & 0 & 0 & 0 & 0 & 0 & 0 & 0 & 0 & $S$ \\
\hline & 0 & 0 & 0 & 0 & 0 & 0 & 0 & 0 & 0 & $k$ \\
\hline \multirow{4}{*}{1} & 4.0 & 2.0 & 2.0 & 1.929 & 1.441 & 1.714 & 1.187 & 1.540 & 1.050 & $\Delta E /|J|$ \\
\hline & 3 & 9 & 3 & 6 & 3 & 6 & 3 & 6 & 3 & $\operatorname{deg}$ \\
\hline & 1 & 1 & 1 & 1 & 1 & 1 & 1 & 1 & 1 & $S$ \\
\hline & 1 & $0,1,2$ & 2 & 2,3 & 3 & 3,4 & 4 & 4,5 & 5 & $k$ \\
\hline \multirow{4}{*}{$\frac{3}{2}$} & 7.5 & 3.5 & 6 & 4.973 & 5.798 & 5.338 & 5.732 & 5.477 & $5.704^{\dagger \dagger}$ & $E_{0} /(N J)$ \\
\hline & 1 & 4 & 1 & 4 & 1 & 4 & 1 & 4 & 1 & $\operatorname{deg}$ \\
\hline & 0 & $1 / 2$ & 0 & $1 / 2$ & 0 & $1 / 2$ & 0 & $1 / 2$ & 0 & $S$ \\
\hline & 1 & 1,2 & 0 & 1,4 & 3 & 2,5 & 0 & 2,7 & 5 & $k$ \\
\hline \multirow{4}{*}{$\frac{3}{2}$} & 4.0 & 3.0 & 2.0 & 2.629 & 1.411 & 2.171 & \begin{tabular}{|c|}
1.117 \\
\end{tabular} & \begin{tabular}{|l|}
1.838 \\
\end{tabular} & $0.938^{\dagger \dagger}$ & $\Delta E /|J|$ \\
\hline & 3 & 16 & 3 & 8 & 3 & 8 & 3 & 8 & 3 & $\operatorname{deg}$ \\
\hline & 1 & $3 / 2$ & 1 & $3 / 2$ & 1 & $3 / 2$ & 1 & $3 / 2$ & 1 & $S$ \\
\hline & 0 & $0,1,2$ & 2 & 2,3 & 0 & 1,6 & 4 & 3,6 & 0 & $k$ \\
\hline \multirow{4}{*}{2} & 12 & 6 & 10 & 8.456 & 9.722 & 9.045 & 9.630 & $9.263^{\dagger \dagger}$ & $9.590^{\dagger \dagger}$ & $E_{0} /(N J)$ \\
\hline & 1 & 1 & 1 & 1 & 1 & 1 & 1 & 1 & 1 & deg \\
\hline & 0 & 0 & 0 & 0 & 0 & 0 & 0 & 0 & 0 & $S$ \\
\hline & 0 & 0 & 0 & 0 & 0 & 0 & 0 & 0 & 0 & $k$ \\
\hline \multirow{4}{*}{2} & 4.0 & 2.0 & 2.0 & 1.922 & 1.394 & 1.652 & 1.091 & $1.431^{\dagger \dagger}$ & $0.906^{\dagger \dagger}$ & $\Delta E /|J|$ \\
\hline & 3 & 9 & 3 & 6 & 3 & 6 & 3 & 6 & 3 & $\operatorname{deg}$ \\
\hline & 1 & 1 & 1 & 1 & 1 & 1 & 1 & 1 & 1 & $S$ \\
\hline & 1 & $0,1,2$ & 2 & 2,3 & 3 & 3,4 & 4 & 4,5 & 5 & $k$ \\
\hline \multirow{4}{*}{$\frac{5}{2}$} & 17.5 & $\begin{array}{r}8.5 \\
\end{array}$ & 15 & 12.434 & 14.645 & \begin{tabular}{|c|}
13.451 \\
\end{tabular} & $14.528^{\dagger}$ & $13.848^{\dagger \dagger}$ & $14.475^{\dagger \dagger}$ & $E_{0} /(N J$ \\
\hline & 1 & 4 & 1 & 4 & 1 & 4 & 1 & 4 & 1 & $\operatorname{deg}$ \\
\hline & 0 & $1 / 2$ & 0 & $1 / 2$ & 0 & $1 / 2$ & 0 & $1 / 2$ & 0 & $S$ \\
\hline & 1 & 1,2 & 0 & 1,4 & 3 & 2,5 & 0 & 2,7 & 5 & $k$ \\
\hline
\end{tabular}

TABLE I. Properties of ground and first excited state of AF Heisenberg rings for various $N$ and $s$ : ground state energy $E_{0}$, gap $\Delta E$, degeneracy deg, total spin $S$ and shift quantum number $k$. $\dagger-$ O. Waldmann, private communication. $\dagger \dagger-$ projection method [36]. Values for higher $N$ are available from the author. 


\section{FIGURES}
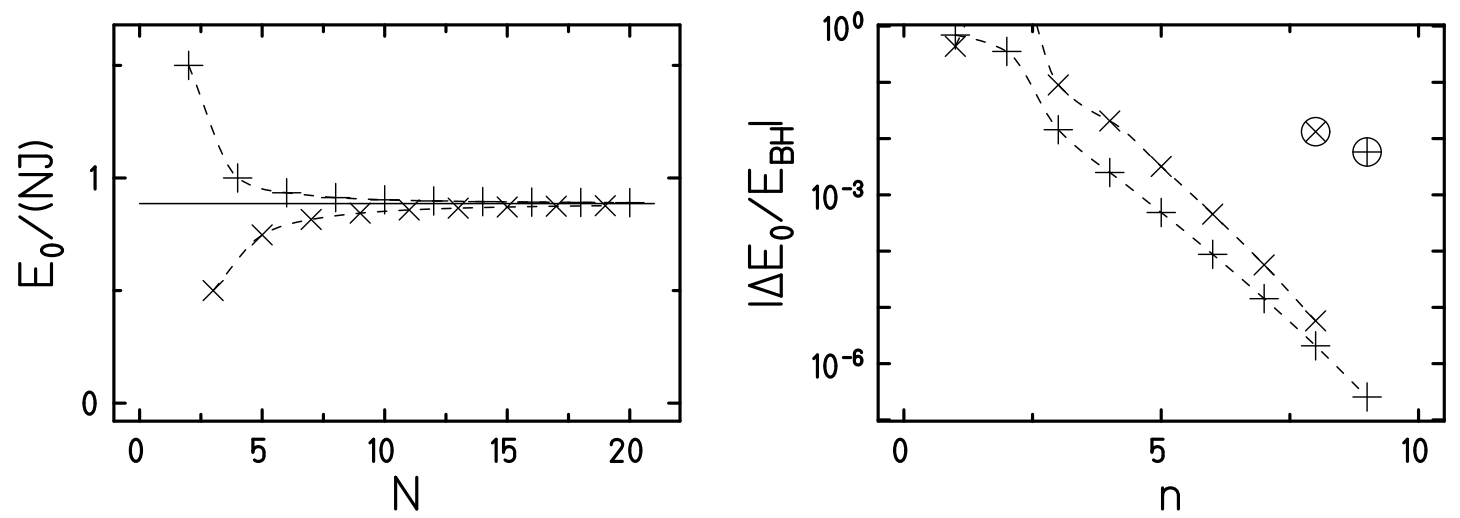

FIG. 1. L.h.s: Ground state energies (symbols) for antiferromagnetic coupled Heisenberg rings of $s=1 / 2$ compared to the large $N$ limit of Bethe and Hulthén (solid line). R.h.s: Relative deviation of the Levin $u$-estimate from the limit of Bethe and Hulthén. Plus symbols are used for even $N$, crosses for odd $N$. The circled symbols show how much the ground state energies itself deviate from the limit.
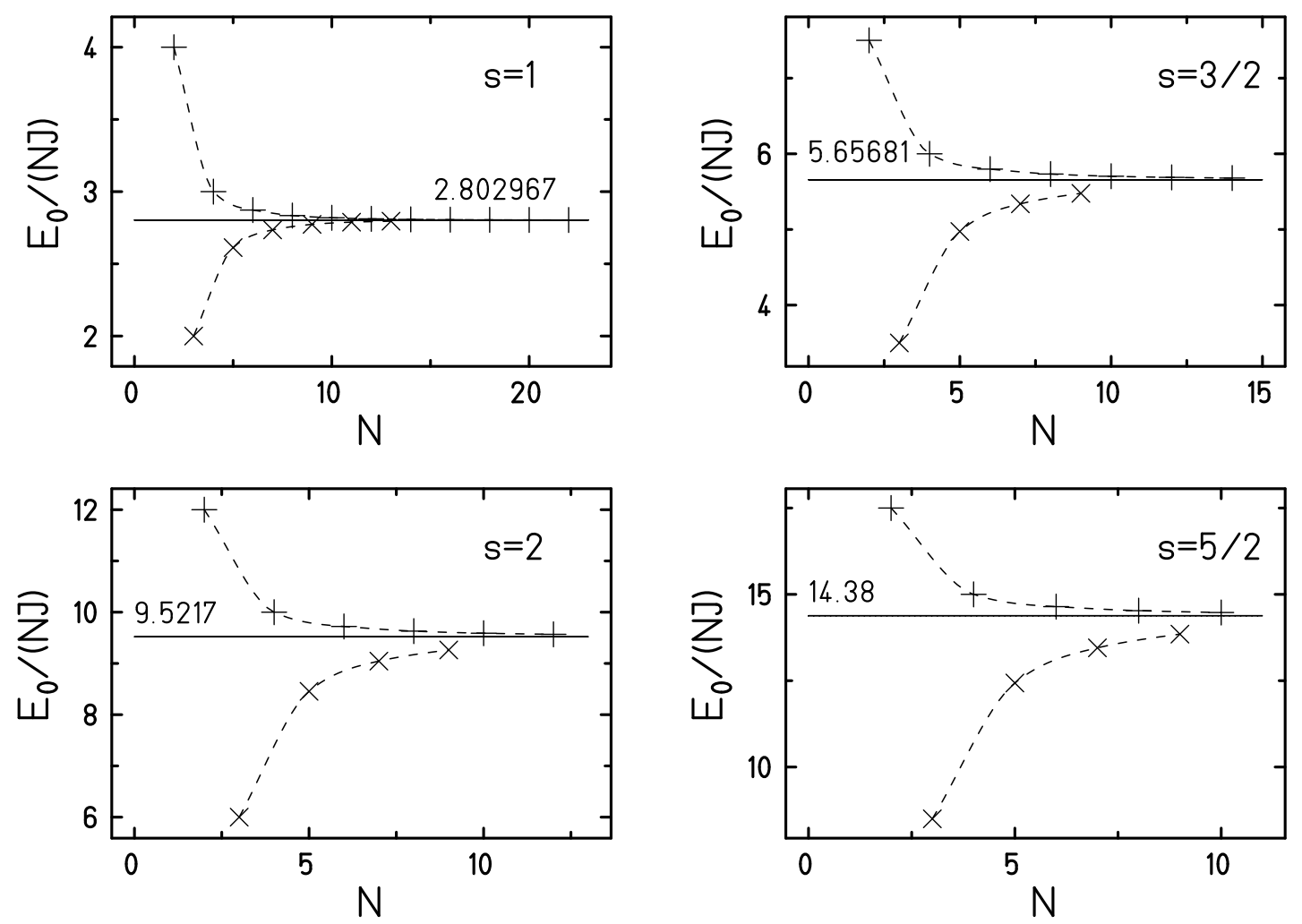

FIG. 2. L.h.s: Ground state energies (symbols) for antiferromagnetic coupled Heisenberg rings compared to the Levin $u$-estimate (hatched area, sometimes shrunk to a line). Plus symbols are used for even $N$, crosses for odd $N$. 

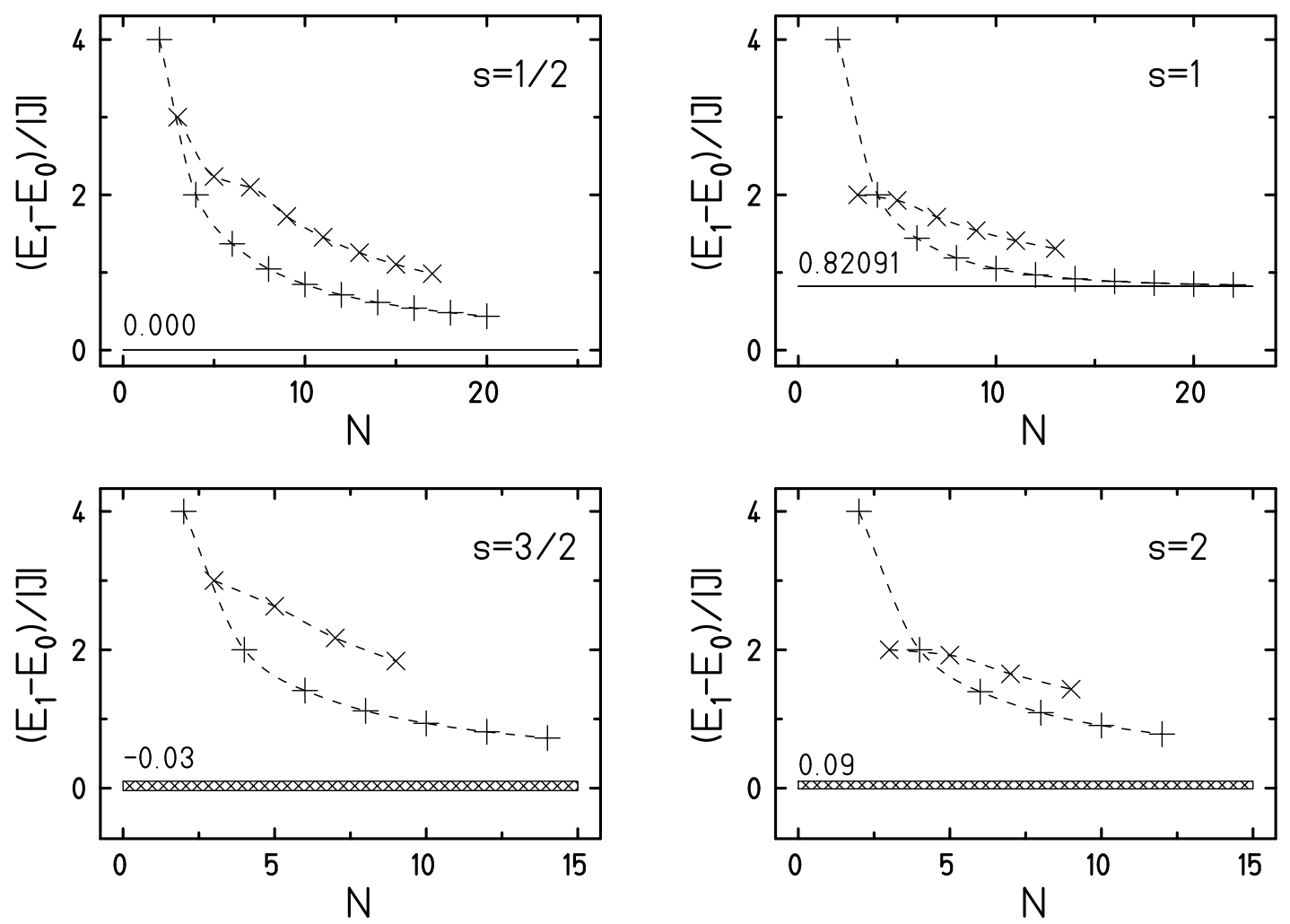

FIG. 3. Excitation energy of the first excited state for antiferromagnetic coupled Heisenberg rings and the Levin $u$-estimate (hatched area). Plus symbols are used for even $N$, crosses for odd $N$. To generate these figures also previously obtained exact diagonalization results of other authors have been used [26 29,31]. 